\title{
DOENÇA DE ALZHEIMER E ESPECTROSCOPIA POR RESSONÂNCIA MAGNÉTICA DO HIPOCAMPO
}

\author{
Eliasz Engelhardt ${ }^{1}$, Denise M. Moreira ${ }^{2}$, Jerson Laks ${ }^{3}$, Valeska M. Marinho ${ }^{4}$, \\ Marcia Rozenthal ${ }^{5}$, Amarino C. Oliveira J ${ }^{6}$
}

\begin{abstract}
RESUMO - Objetivos: Obtenção de dados do espectro de metabolitos por ressonância magnética da formação hipocampal no idoso normal e com doença de Alzheimer (DA). M étodo: Os indivíduos foram pareados por idade, sendo 20 na amostra normal, CDR=0 e 40 com DA 3 CDR=1 e2. Utilizou-se aparelho Signa Horizon LX-

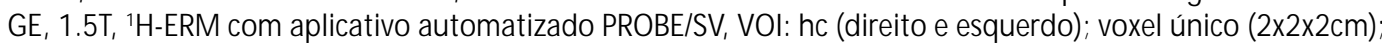
TR 1500ms/TE 50ms; PRESS; metabolitos: $\mathrm{N}$-acetilaspartato (Naa), colina (Cho), creatina (Cr), mio-inositol (ml). Resultados: Os presentes dados se referem aos quocientes de Naa, Cho e ml, com $\mathrm{Cr}$ tomada como referência e relação $\mathrm{ml} / \mathrm{Naa}$. O estudo mostrou o Naa reduzido, o $\mathrm{ml}$ e a relação $\mathrm{ml} / \mathrm{Naa}$ aumentados e os resultados em relação à Cho foram variados. Os resultados da amostra global dos pacientes com DA em comparação à média $\pm d p$ da amostra normal foram significativos para Naa, $\mathrm{ml}$ e ml/Naa $(p<0,01)$. A precisão, tomando os valores de modo individual das duas amostras, mostrou sensibilidade, especificidade e valor preditivo positivo satisfatórios. Conclusão: Os presentes resultados podem ser usados como ferramenta útil para detectar alterações patológicas no hipocampo de pacientes com DA, permitindo diagnóstico de maior precisão e mais precoce da doença.
\end{abstract}

PALAVRAS-CHAVE: ressonância magnética, espectroscopia de prótons, hipocampo, doença de Alzheimer.

\begin{abstract}
Alzheimer's disease and magnetic resonance spectroscopy of the hippocampus
ABSTRACT - Objective: Acquisition of data of magnetic resonance metabolite spectrum of the hippocampal formation (hippocampus-hc) in the elderly, normal and with Alzheimer's disease (AD). Method: Subjects matched for age: a. normal sample $(n=20), C D R=0$, and $b$. AD sample $(n=40), C D R 1$ and 2. Technique: Signa Horizon LX-GE, 1.5T, ${ }^{1} \mathrm{H}-\mathrm{MRS}$ with automated software PROBE/SV, VOI: hc (right and left); single voxel ( $\left.2 \times 2 \times 2 \mathrm{~cm}\right)$; TR 1500ms/TE 50ms; PRESS; metabolites: N-acetylaspartate (Naa), choline (Cho), creatine (Cr), myo-inositol (ml). Results: The present data relate to the ratios of $\mathrm{Naa}$, Cho and $\mathrm{ml}$, with $\mathrm{Cr}$ taken as reference, and the $\mathrm{ml} /$ Naa ratio. The study showed reduction of Naa, increase of $\mathrm{ml}$ and of the $\mathrm{ml} / \mathrm{Naa}$ ratio, and not consistent results for Cho. The results of the whole sample of AD patients compared to the pooled normal mean $\pm \mathrm{sd}$ were significant for $\mathrm{Naa}, \mathrm{ml}$ and $\mathrm{ml} / \mathrm{Naa}(\mathrm{p}<0.01)$. Accuracy in relation to the individual values of both samples showed satisfactory levels of sensitivity, specificity and positive predictive value. Conclusion: The present results can be used as a helpful tool to detect pathologic changes of the hippocampus in $A D$, and allowing greater accuracy and an earlier diagnosis of this disease.
\end{abstract}

KEY WORDS: magnetic resonance, proton spectroscopy, hippocampus, Alzheimer's disease.

A doença de Alzheimer (DA) éa causa da demência mais prevalente tanto no grupo etário pré-senil quanto no senil, observando-se seu aumento gradual como envelhecimento. As manifestações características compreendem comprometimento cognitivo, sintomas psicológicos e do comportamento, sinais neurológicos e declínio nas atividades de vida diária, de um modo progressivo. O seu diagnóstico é baseado em história clínica sugestiva, utilização de critérios sistematizados (DSM-IV, NINCDS-ADRDA), exames laboratoriais e de neuroimagem, permitindo finalmente, em vida, o diagnóstico mais próximo do correto que é de "doença de Alzheimer provável" ${ }^{1 .}$

Estudo realizado no Setor de Neurologia Cognitiva e do Comportamento do Instituto de Neurologia Deolindo Couto (INDC) da Universidade Federal do Rio de Janeiro (UFRJ), Centro de Atendimento para Pessoas com Doença de Alzheimer (CDA) do IPUB da Universidade

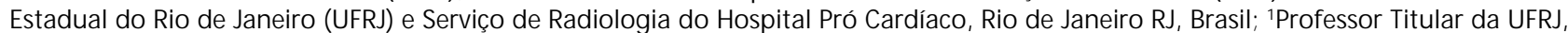
Coordenador do Setor de NCC do INDC/UFR; ; ${ }^{2}$ Professor Adjunto da UFR, responsável pelo Setor de Radiologia, INDC/UFR]; ${ }^{3}$ Professor Adjunto da UERJ, Professor da Faculdade de Medicina da UNESA, Diretor Técnico do CDA, IPUB/UFRJ; ${ }^{4}$ Mestre em Psiquiatria, IPUB/UFR, médico do CDA, IPUB/UFR;; ${ }^{5}$ Doutor em Psiquiatria, IPUB/UFR, Professor da Faculdade de Medicina da UNESA; ${ }^{6}$ Chefe do Setor de Radiologia, Hospital PróCardíaco e INCA.

Recebido 18 Junho 2001, recebido na forma final 30 Julho 2001. Aceito 3 Agosto 2001.

Dr. Eliasz Engelhardt - Av. N.S. Copacabana, 749/1101 - 22050-000 Rio de Janeiro RJ - Brasil. 
Entre os recursos frequentemente utilizados para reforçar o diagnóstico da DA estão os exames de neuroimagem estrutural (por exemplo, volumetria do hipocampo) e os de neuroimagem funcional (por exemplo, espectroscopia por ressonância magnética/ERM).

A espectroscopia de prótons por ressonância magnética ( ${ }^{1} \mathrm{H}$-ERM), no estudo de DA, foi introduzida por Shiino et al. ${ }^{2}$, que relataram diminuição de $\mathrm{N}$-acetilaspartato (Naa), considerado marcador de integridade neuronal, em pacientes com demência degenerativa primária. A partir dessa data, a ERM passou a ser frequentemente utilizada em termos de exame complementar para reforçar o diagnóstico. Miller et $\mathrm{al}^{3}$. relataram o aumento também de outro metabolito, o mio-inositol $(\mathrm{ml})$, pertencente à via metabólica dos inositídeos, além de marcador de neuroglia. O binômio diminuição de Naa-aumento de ml passou a ser considerado característico da DA. Variações da colina (Cho) também foram encontradas, porém de modo menos consistente, refletindo metabolismo de membrana de células gliais relacio- nadas às áreas de degeneração neuronal. Finalmente deve ser mencionada a creatina ( $\mathrm{Cr}$ ), relacionada com atividade metabólica energética e que se mantém relativamente estável até fases muito adiantadas do processo degenerativo, servindo assim como referência interna para estabelecer relações (quocientes) com os outros metabolitos ${ }^{4-6}$. O índice $\mathrm{ml} / \mathrm{Naa}$ foi proposto por Schonk et al. ${ }^{7}$, para dar maior sensibilidade aos resultados obtidos. Um número relativamente grande de estudos em pacientes com DA demonstrou redução de Naa, aumento de $\mathrm{ml}$ e da relação $\mathrm{ml} / \mathrm{Naa}$ e eventualmente aumento da Cho em áreas corticais associativas posteriores (Tabela 1). Esses estudos foram realizados com ERM gráfico (ERMg) ou com ERM de imagem (ERMi) ${ }^{4,5,8}$.

Estudos encontrados visando áreas límbicas são poucos (Tabela 1). Assim, Kantarci et al. ${ }^{9}$ examinaram a parte posterior do giro do cíngulo, com achados gerais semelhantes aos acima referidos, sugerindo ainda uma cronologia das variações, com aumento de $\mathrm{ml}$ em fases mais iniciais, diminuição de Naa e aumento de Cho em fases mais adiantadas da

Tabela 1. Principais estudos de ${ }^{1} \mathrm{H}$-ERM : áreas estudadas, resultados obtidos, principais autores.

\begin{tabular}{|c|c|c|c|c|}
\hline \multicolumn{2}{|c|}{ Áreas estudadas } & \multicolumn{2}{|c|}{ Variação de metabolitos } & \multirow{2}{*}{$\begin{array}{l}\text { Principais autores } \\
\text { Shiino et al., 1993² }\end{array}$} \\
\hline \multirow{11}{*}{\multicolumn{2}{|c|}{ Associativas }} & redução & Naa & \\
\hline & & redução & Naa & \\
\hline & & aumento & $\mathrm{ml}$ & Miller et al., $1993^{3}$ \\
\hline & & redução & Naa & Meyerhoff et al., $1994^{17}$ \\
\hline & & aumento & Cho & Pfefferbaum et al., $1999^{24}$ \\
\hline & & redução & Naa & \\
\hline & & aumento & $\mathrm{ml}$ & \\
\hline & & aumento & $\mathrm{ml} / \mathrm{Naa}$ & Shonk et al., $1995^{7}$ \\
\hline & & redução & Naa & \\
\hline & & aumento & Cho & \\
\hline & & aumento & $\mathrm{ml}$ & Lazeyras et al., $1998^{25}$ \\
\hline \multirow[t]{11}{*}{ Límbicas } & cíngulo & redução & Naa & \\
\hline & & aumento & $\mathrm{ml}$ & \\
\hline & & aumento & Cho & Kantarci et al., $2000^{9}$ \\
\hline & hipocampo & redução & Naa & \\
\hline & & aumento & Cho & Block et al., $1995^{10}$ \\
\hline & & redução & Naa & Schuff et al., $1997^{11}$ \\
\hline & & variado & Cho & Jessen et al., $2000^{12}$ \\
\hline & & redução & Naa & \\
\hline & & aumento & $\mathrm{ml}$ & \\
\hline & & aumento & Cho & \\
\hline & & aumento & $\mathrm{ml} / \mathrm{Naa}$ & Engelhardt et al., $2000^{14}$ \\
\hline
\end{tabular}


evolução. Até recentemente, apenas dois grupos relataram resultados de ERMi do hipocampo (formação hipocampal), o de Block e o de Schuff. O primeiro centrou o estudo na Cho, cujo ciclo inclui o metabolismo da acetilcolina ${ }^{10}$. O segundo estudou o Naa e Cho. Outro trabalho do grupo de Block foi com ERMg, mostrando resultados semelhantes aos de Shuff ${ }^{11}$. Não foram obtidos, nos trabalhos citados, resultados do $\mathrm{ml}$, já que o tempo de eco utilizado foi alto. Desse modo não foi considerado o ml, aumentado frequentemente na DA e apontado como importante no diagnóstico, assim como o índice ml/ $\mathrm{Naa}^{3,6}$. Os estudos de Jessen et al. ${ }^{12}$ e os preliminares de Engelhardt et al. ${ }^{13,14}$ foram realizados com equipamento e aplicativo que permitem exames em situação clínica. Os dados obtidos em relação ao Naa, Cho e $\mathrm{Cr}$ nesses estudos apresentaram padrão geral semelhante aos relatados para áreas associativas (diminuição de Naa e variação de Cho). Os dados relacionados ao $\mathrm{ml}$ e consequentemente à relação $\mathrm{ml} / \mathrm{Naa}$, índices considerados importantes para o diagnóstico da DA, foram obtidos apenas por EngeIhardt et al. ${ }^{13,14}$, mostrando-se elevados em número significativo de pacientes.

Lembrando que o processo degenerativo na DA tem início em áreas límbicas da região temporal medial (formação hipocampal, região rinal) e se correlaciona com as manifestações clínicas iniciais (comprometimento de memória) ${ }^{15,16}$, pode-se considerar que o exame dessa região deve mostrar alterações em fase mais precoce que as obtidas em áreas associativas. Grande número de estudos consideraram o hipocampo do ponto de vista estrutural, procurando reconhecer medidas indicativas de sua atrofia. A perda neuronal, no entanto, decorrente do processo degenerativo, é frequentemente acompanhada por gliose reativa que atenua a atrofia tissular. Assim, apenas os achados volumétricos podem subestimar a extensão detal perda. Por outro lado, a espectroscopia, técnica sensível a variações de metabolitos, poderia se constituir em marcador mais específico de perda neuronal em comparação à atrofia observada pela neuroimagem estrutural11,17,18. A utilização de tal técnica, tendo como alvo o hipocampo, pode reforçar a precisão diagnóstica e permitir o seu estabelecimento mais precoce na DA.

\section{MÉTODO}

Sujeitos. Foram estudadas duas amostras: a) normal: $\mathrm{n}=20(\mathrm{~m}=10 / \mathrm{f}=10)$; idade: 73,5 $\pm 5,0$ (66 a 86) anos; sem queixas cognitivas ou psiquiátricas; mini-exame do estado mental (MEEM): 28,6 1.0 (27 a 30); estágio clinical dementia rating $(C D R)=0 ; b)$ DA provável (critérios da DSM-IV e da NINCDS-ADRDA): $\mathrm{n}=40(\mathrm{~m}=18 / \mathrm{f}=22)$; ida-

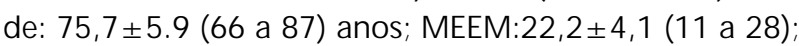
escore isquêmico (Hachinski) <4 (0-3); estágio: CDR 1 e 2.

Técnicas. Foi utilizado aparelho Signa Horizon LX-GE, 1.5T, obtenção de espectro de prótons ( ${ }^{1} \mathrm{H}$-ERM) com ajuda de aplicativo PROBE/SV ${ }^{19,20}$; gráfico de metabolitos evidenciando N-acetilaspartato, colina, creatina, mio-inositol; região de interesse: hipocampos (direito e esquerdo); voxel único de $2 \times 2 \times 2 \mathrm{~cm}$ (8 ml); tempo de repetição (TR) 1500 $\mathrm{ms} /$ tempo de eco (TE) 50 ms; PRESS.

Estatística. Foram realizados cálculos básicos de média, desvio-padrão, teste de significância, teste de precisão para os valores obtidos ${ }^{21}$.

Ética. O protocolo referente à amostra normal foi aprovado pela Comissão de Ética (CEP-IPUB/UFRJ); a amostra com DA foi constituída a partir de casos avaliados em situação clínica de rotina.

\section{RESULTADOS}

Os dados obtidos se referem aos valores da amostra com DA em comparação à normal, considerando-se as relações de Naa, Cho e $\mathrm{ml}$ com $\mathrm{Cr}$ tomado como referência interna, além da relação $\mathrm{ml} / \mathrm{Naa}$.

Os resultados obtidos são expressos de duas maneiras: globais e individuais.

a. globais - considerando que não foi encontrada diferença estatística significativa entre os valores dos hipocampos direito e esquerdo, assim como feminino e masculino, os resultados globais serão considerados aos dados reunidos da amostra inteira, normal em comparação à patológica (Tabela 2).

Tabela 2. Valores das relações de metabolitos obtidos a partir dos dados reunidos, em sujeitos normais e com DA provável (hipocampos direito e esquerdo, masculino e feminino).

\begin{tabular}{lcccc}
\hline & $\mathrm{Naa} / \mathrm{Cr}$ & $\mathrm{ml} / \mathrm{Cr}$ & $\mathrm{Cho} / \mathrm{Cr}$ & Cho/Naa \\
\hline Normal & $1.46 \pm 0.14$ & $0.51 \pm 0.12$ & $0.95 \pm 0.14$ & $0.36 \pm 0.09$ \\
DA prwel & $1.33 \pm 0.18$ & $0.60 \pm 0.12$ & $0.94 \pm 0.12$ & $0.47 \pm 0.09$ \\
Teste t & $\alpha=0.01$ & $\alpha=0.01$ & ns & $\alpha=0.01$ \\
\hline prwel, provável; teste t de significância entre os normais e os patológicos $(\mathrm{p}<0.01)$.
\end{tabular}


Tabela 3. Teste de precisão dos valores dos metabolitos obtidos em uma amostra de pacientes com DA $(n=40)$ em comparação a sujeitos normais $(n=20)$.

\begin{tabular}{lcccc}
\hline Metblto & $\mathrm{dp}$ & $\mathrm{s}$ & $\mathrm{e}$ & $\mathrm{vp}+$ \\
\hline $\mathrm{Naa} / \mathrm{Cr}$ & -1 & 0.80 & 0.75 & 0.80 \\
$\mathrm{nN}=20^{*}$ & -1.5 & 0.60 & 0.95 & 0.96 \\
$\mathrm{nDA}=40^{* *}$ & -2 & 0.35 & 0.95 & 0.93 \\
$\mathrm{ml} / \mathrm{Cr}$ & +1 & 0.42 & 0.82 & 0.82 \\
$\mathrm{nN}=17^{*}$ & +1.5 & 0.27 & 0.82 & 0.75 \\
$\mathrm{nDA}=33^{* *}$ & +2 & 0.15 & 0.88 & 0.71 \\
$\mathrm{Cho} / \mathrm{Cr}$ & +1 & 0.17 & 0.70 & 0.53 \\
$\mathrm{nN}=20^{*}$ & +1.5 & 0.05 & 0.85 & 0.40 \\
$\mathrm{nDA}=39^{* *}$ & +2 & 0.02 & 0.95 & 0.50 \\
$\mathrm{ml} / \mathrm{Naa}$ & +1 & 0.65 & 0.70 & 0.80 \\
$\mathrm{nN}=17 *$ & +1.5 & 0.50 & 0.82 & 0.84 \\
$\mathrm{nDA}=32 * *$ & +2 & 0.31 & 0.90 & 0.83 \\
\hline
\end{tabular}

Metblto, metabolitos (quocientes); dp, desvio padrão; s, sensibilidade; e, especificidade; $v p+$, valor preditivo positivo. *valores encontrados normais; **valores encontrados com DA.

b. individuais - foram calculadas a precisão dos valores obtidos (sensibilidade, especificidade e valor preditivo positivo), expressos na Tabela 3 e os percentuais para cada parâmetro referente a um desvio padrão (dp), mostrados na Tabela 4.

\section{DISCUSSÃO}

A prevalência crescente de pacientes com DA, as possibilidades terapêuticas existentes e as novas, que vêm surgindo, levam à necessidade de diagnóstico mais precoce e preciso da doença. $A^{1} \mathrm{H}$-ERM do cérebro pode ser considerada instrumento valioso de pes- quisa e clínica por permitir conhecer a composição de metabolitos de amostra(s) de tecido nervoso normal ou patológico in vivo e de modo não-invasivo, permitindo uma compreensão dos mecanismos fisiológicos e fisiopatológicos em condições normais e na doença22.

A importância de focalizar o estudo no hipocampo decorre da sequência da progressão patológica da doença de Alzheimer. Assim a degeneração tem início em áreas límbicas da região medial do lobo temporal (córtex entorrinal e formação hipocampal), progredindo para as áreas associativas temporoparietais e frontais, as áreas primárias sendo acometidas apenas nos estágios mais avançados ${ }^{15}$. Desse modo, ao se visar o diagnóstico precoce, aspecto dos mais desejáveis para intervenções terapêuticas igualmente precoces, o foco do exame deve ser dirigido para a região hipocampal, inicialmente atingida pelo processo degenerativo. Considerando que poucos estudos com foco no hipocampo foram encontrados na literatura ${ }^{10-12}$, possivelmente pelas dificuldades técnicas envolvidas no estudo dessa região, os dados apresentados no presente estudo poderão ser de valia para ajudar na detecção de alterações precoces na DA. Os dados comparativos entre os diversos estudos visando o hipocampo se encontram na Tabela 5.

O estudo de Block et al. ${ }^{10}$ mostrou que 7/12 (58\%) pacientes apresentaram redução de Naa em comparação a um grupo controle. Encontraram ainda um aumento significativo de Cho, o que não foi verificado em novo estudo do grupo ${ }^{12}$. O trabalho de Schuff et al. ${ }^{11}$ mostrou que o Naa hipocampal permitiu classificar corretamente $80 \%$ dos pacientes

Tabela 4. Relação entre casos positivos e o número de casos e percentual encontrados para $1 \mathrm{dp}$ para os diversos quocientes de metabolitos. Positividade (positiv) se refere ao número de casos positivos em pelo menos um parâmetro ( $\mathrm{Naa} / \mathrm{Cr}, \mathrm{ml} / \mathrm{Cr}, \mathrm{ml} / \mathrm{Naa}$ ) por total.

\begin{tabular}{lcccccc}
\hline & $\mathrm{Naa} / \mathrm{Cr}$ & $\mathrm{ml} / \mathrm{Cr}$ & \multicolumn{2}{c}{$\mathrm{Cho} / \mathrm{Cr}$} & $\mathrm{ml} / \mathrm{Naa}$ & positiv \\
\hline $\mathrm{dp}$ & -1 & +1 & +1 & -1 & +1 & \\
Normal & & & & & & \\
$\mathrm{x} / \mathrm{n}$ & $5 / 20$ & $3 / 17$ & $6 / 20$ & $4 / 20$ & $5 / 17$ & $9 / 20$ \\
$\%$ & 25 & 17 & 30 & 20 & 39 & 55 \\
$\mathrm{DA}$ & & & & & & \\
$\mathrm{y} / \mathrm{n}$ & $32 / 40$ & $14 / 33$ & $7 / 39$ & $5 / 39$ & $21 / 32$ & $36 / 40$ \\
$\%$ & 80 & 42 & 18 & 12 & 65 & 90 \\
& & & & & & \\
\hline
\end{tabular}

$x / n$, normal; $y / n, D A$. 
Tabela 5. Estudos do hipocampo - dados comparativos dos estudos realizados.

\begin{tabular}{lccccccc}
\hline Autores & $\begin{array}{c}\text { DA } \\
\text { n/idade }\end{array}$ & $\begin{array}{c}\text { Controle } \\
\text { n/idade }\end{array}$ & ERM & Naa & ml & Cho & ml/Naa \\
\hline Block et al., 1994 & $12 / 48-78$ & $17 / 30-73$ & imagem & redzdo & - & aumtdo & - \\
Schuff et al., 1997 & $12 / 55-82$ & $17 / \mathrm{nd}$ & imagem & redzdo & - & $\mathrm{ns}$ & - \\
Jessen et al., 2000 & $20 / 69.8 \pm 8.1$ & $18 / 65.6 \pm 7.8$ & gr-vx17.5 & redzdo & - & redzdo & - \\
Engelhardt et al., 2001 & $40 / 75.7 \pm 5.9$ & $20 / 73.5 \pm 5.0$ & gr-vx08.0 & redzdo & aumtdo & ns & aumtdo \\
\hline
\end{tabular}

gr-vx08.0, gráfico-voxel de 8.0ml; gr-vx17.5, gráfico-voxel de 17.5ml; nd, não descrito; redzdo, reduzido; aumtdo, aumentado; ns, não significativo.

com DA e $75 \%$ dos sujeitos controle. O presente estudo mostrou redução de Naa em 32/40 (80\%) dos pacientes, aumento de ml em 14/33 (42\%) e o índice $\mathrm{ml} / \mathrm{Naa}$ encontrou-se aumentado em 21/32 (65\%). A positividade em 1 ou mais desses 3 parâmetros chegou a 36/40 (90\%) pacientes, índice que pode ser considerado elevado. Foi possível ainda calcular teste de precisão dos valores dos metabolitos, encontrando-se na Tabela 3 os índices de sensibilidade, especificidade e valor preditivo positivo para 1, 1.5 e $2 \mathrm{dp}$. Pode-se considerar, de modo geral, que os melhores índices correspondem aos encontrado para $1 \mathrm{dp}$, podendo essas cifras serem consideradas satisfatórias. Esse aspecto é coerente considerando a margem estreita de variação dos metabolitos, tanto em condições normais, como em patológicas.

Finalmente, deve-se considerar a presença de superposição de valores (DA vs normal), observado pela maioria dos autores que estudaram a questão e também no presente estudo. Deve-se certamente à limitada variação dos metabolitos nas diversas condições, normais e patológicas, como já assinalado. A procura de um marcador biológico, sem ser a verificação neuropatológica (biópsia de tecido cerebral, obtida em condições excepcionais) para confirmar o diagnóstico, tem sido exaustiva, porém até agora não encontrado. Entretanto, lançam-se mão de exames especializados que permitem refinar o diagnóstico e torná-lo "mais provável". Para tanto podem ser utilizados "marcadores substitutos" (bioquímicos, neuroimagem) ${ }^{23}$. A ERM é exame de neuroimagem que pode ser um bom candidato como marcador substituto e os seus resultados encontrados para o hipocampo podem ser considerados importantes para refinar o diagnóstico da DA.

\section{CONCLUSÃO}

A amostra de pacientes com DA em comparação a um grupo controle caracterizou-se pela redução de Naa, aumento de $\mathrm{ml}$ e da relação $\mathrm{ml} / \mathrm{Naa}$, en- quanto os valores em relação à Cho foram variáveis.

Os valores da amostra patológica mostraram-se significativos, tanto de modo global, como individual, o que permite a utilização desses resultados, principalmente conjugados, para aumentar a precisão do diagnóstico e para obtê-lo de modo mais precoce, considerando as características da região estudada.

A ${ }^{1} \mathrm{H}$-ERM do hipocampo pode portanto ser considerada um instrumento importante por permitir conhecer a composição de metabolitos de amostra(s) de tecido nervoso normal e patológico in vivo e de modo não invasivo, permitindo uma melhor compreensão dos mecanismos fisiológicos e fisiopatológicos com objetivos clínicos e de pesquisa.

\section{REFERÊNCIAS}

1. McKhann G, Drachman D, Folstein M, Katzman R, Price D, Stadlan EM. Clinical diagnosis of Alzheimer's disease: report of the NINCDSADRDA Work Group under the auspices of Department of Health and Human Services Task Force on Alzheimer's disease. Neurology 1984;34:939-944.

2. Shiino A, Matsuda M, Morikawa S, Inubushi T, A kiguchi I, Handa J. Proton magnetic resonance spectroscopy with dementia. Surg N eurol 1993; 39:143-147.

3. Miller BL, Moats RA, Shonk T, Ernst T, Woolley S, Ross BD. Alzheimer disease: depiction of increase cerebral myo-inositol with proton $\mathrm{mr}$ spectroscopy. Radiology 1993;187:433-437.

4. Engelhardt E, Moreira DM, Laks J, Rozenthal M. Espectroscopia de prótons $(1 \mathrm{H})$ por ressonância magnética, neuroquímica cerebral ediagnóstico de doenças neurológicas. Rev Bras Neurol 2000; 36:11-25.

5. Valenzuela MJ, Perminder S. Magnetic resonance spectroscopy in AD. Neurology 2001;56:592-598.

6. Ross BD, Bluml S. Magnetic Resonance spectroscopy of the human brain. Anat Rec (New Anat) 2001;265:54-84.

7. Schonk TK, Moats RA, Gifford P et al. Probable alzheimer's disease: diagnosis with proton $\mathrm{mr}$ spectroscopy. Radiology 1995;195:65-72.

8. Ross BD, Bluml S, Cowan R et al. In vivo MR spectroscopy of human dementia. Neuroimaging Clin N Am 1998;8:809-822.

9. Kantarci K, Jack CR Jr, Xu YC, et al. Regional metabolic patterns in mild cognitive impairment and Alzheimer's disease: a 1H MRS study. Neurology 2000; 55:210-217.

10. Block W, Träber F, Kuhl CK et al. IH-MR-spektroscopischeBildgebung bei Patienten mit Klinisch gesichertem Morbus Alzheimer. Fortschr Röntgenstr 1995;163:230-237.

11. Schuff N, Amend D, Ezekiel BA, et al. Changes of hippocampal Nacetyl aspartate and volume in Alzheimer's disease: a proton MR spectroscopic imaging and MRI study. Neurology 1997;49:1513-1521.

12. Jessen $F$, Block W, Träber F, et al. Proton MR spectroscopy detects a relative decrease of $\mathrm{N}$-acetylaspartate in the medial temporal lobe of patients with AD. Neurology 2000;55:684-688. 
13. Engelhardt E, Moreira DM, Laks J, Marinho VM, Rozenthal M, Oliveirajr AC. Proton magnetic resonancespectroscopy of thehippocampus: normatization in the elderly. Abstracts - World Alzheimer Congress (2000). Neurobiol Aging 2000;21(Suppl):S41.

14. Engelhardt E, Moreira DM, Laks J et al. Proton magnetic resonance spectroscopy of the hippocampus in Alzheimer's disease Abstracts - Joint Meeting-International PsychogeriatricAssociation and Brazilian Association of Geriatric Neuropsychiatry -JBNPG 2000;edição especial:43.

15. Braak H, Braak E. Neuropathological staging of Alzheimer-related changes. Acta Neuropathol 1991;82:239-259.

16. Grober E, Dickson D, Sliwinski MJ et al Memory and mental status correlates of modified Braak staging. Neurobiol A ging 1999;20:573-579.

17. Meyerhoff DJ, Mackay S, Constans JM et al. Axonal injury and membrane alterations in alzheimer's disease suggested by in vivo proton magnetic resonance spectroscopic imaging. Ann Neurol 1994;36:40-47.

18. Schuff $N$, Amend D, Meyerhoff DJ et al. Alzheimer's disease: quantitative $\mathrm{H}-1 \mathrm{MR}$ spectroscopic imaging of frontoparietal brain. Radiology 1998;207:91-102.
19. Kohler S. Signa advantageapplicattions guide. PROBE/ SV. GE Medical Systems. Milwaukee: General Electric Co, 1993.

20. Moats RA, Shonk T. Evaluation of automated MR spectroscopy: application in alzheimer disease. AJNR 1995;16:1779-1782.

21. Dawson-Saunders B, Trapp RG. Basic and clinical biostatistics. Norwalk:A ppleton \& Lange, 1990.

22. Danielsen ER, Ross B. Magnetic resonance spectroscopy diagnosis of neurological diseases. New York:Marcel Dekker, 1999.

23. Blennow K, Vanmechelen E. Combination of the different biological markers for increasing specificity of in vivo A lzheimer'stesting.J Neural Transmis 1998;53(Suppl):223-235.

24. Pfefferbaum A, A dalsteinsson E, Spielman D, Sullivan EV, Lim KO. In vivo brain concentrations of $n$-acetyl compounds, creatine, and choline in Alzheimer disease. Arch Gen Psychiatry 1999;56:185-192.

25. Lazeyras F, Charles HC, Tuples LA, et al. Metabolic brain mapping in Alzheimer's disease using proton magnetic resonance spectroscopy. Psychiatry Res: Neuroim Sec 1998;82:95-106. 